\title{
Fluoroscopically guided laser lithotripsy versus extracorporeal shock wave lithotripsy for retained bile duct stones: a prospective randomised study
}

\author{
R Jakobs, H E Adamek, M Maier, M Krömer, C Benz, W R Martin, J F Riemann
}

\begin{abstract}
Background and aims-To compare extracorporeal shock wave lithotripsy (ESWL) and laser induced shock wave lithotripsy (LISL) of retained bile duct stones to stone free rate, number of therapeutic sessions, and costs.

Patients-Thirty four patients were randomly assigned to either ESWL or LISL therapy. The main reasons for failure of standard endoscopy were due to stone impaction $(n=12)$, biliary stricture $(n=8)$, or large stone diameter $(n=14)$.

Methods-An extracorporeal piezoelectric lithotripter with ultrasonic guidance and a rhodamine 6G laser with an integrated stone tissue detection system were used. LISL was performed exclusively under radiological control.

Results-Using the initial methods complete stone fragmentation was achieved in nine of 17 patients $(52 \cdot 4 \%)$ of the ESWL group and in 14 of 17 patients $(82.4 \%)$ in the LISL group, or combined with additional fragmentation techniques 31 of the 34 patients $(91 \cdot 2 \%)$ were stone free at the end of treatment. In comparison LISL tended to be more efficient in clearing the bile ducts $(p=0 \cdot 07, N S)$. Significantly less fragmentation sessions (1.29 $v$ 2.82; $p=0.0001)$ and less additional endoscopic sessions $(0.65 v 1.6 ; p=0.002)$ were necessary in the LISL group. There were no major complications in either procedure. Conclusions-Compared with ESWL, fluoroscopically guided LISL achieves stone disintegration more rapidly and with significantly less treatment sessions, which leads to a significant reduction in cost. (Gut 1997; 40: 678-682)
\end{abstract}

Medicine C, Klinikum

Ludwigshafen,

Academic Teaching

Hospital of the

University of Mainz,

Ludwigshafen,

Germany

R Jakobs

H E Adamek

M Maier

M Krömer

C Benz

W R Martin

J F Riemann

Correspondence to:

Dr Ralf Jakobs,

Department of Medicine C,

Klinikum Ludwigshafen,

Klinikum Ludwigshafen,

Bremserstrasse 79, D-67063
Ludwigshafen, Germany.

Accepted for publication

23 January 1997
Keywords: laser lithotripsy, extracorporeal shock wave lithotripsy, retained bile duct stones, endoscopic retrograde cholangiopancreatography.

Since the introduction of endoscopic sphincterotomy in $1974,{ }^{1}{ }^{2}$ endoscopy has been widely used in the treatment of common bile duct stones. Particularly in elderly patients, after previous cholecystectomy and before laparoscopic cholecystectomy, endoscopic bile duct clearance is preferred. ${ }^{3}$ Successful removal of bile duct stones by standard endoscopy was achieved in $85 \%$ to $95 \%$ of patients in several series. ${ }^{4}$ For the remaining $5 \%$ to $15 \%$ of stones mechanical lithotripsy is the first line treatment with a therapeutic success of about $80 \%$ to $97 \%$ and the best reported cost effectiveness. This method fails in a few patients because the calculi are too large, impacted, or located above a biliary stricture. ${ }^{5}$

Biliary stones are resistant to the above methods in only $2 \%$ to $5 \%$ of patients. For this selected group extracorporeal and various intracorporeal lithotripsy procedures were developed over the past decade. ${ }^{6-8}$ Laser lithotripsy is the latest development in the field of fragmentation techniques. A major problem in using this device is the requirement of cholangioscopic guidance, which is expensive due to the cost of the cumbersome endoscopic equipment and the need for two experienced endoscopists operating the "mother and baby" endoscope. ${ }^{9}$ The most promising laser lithotripter has an integrated stone tissue detection system, ${ }^{10}$ which can be used under sole fluoroscopic guidance and thereby should reduce the expense of this means of fragmentation.

The aim of our prospective trial was to compare ultrasonically guided extracorporeal shock wave lithotripsy (ESWL) with laser induced intracorporeal lithotripsy (LISL) performed under pure radiological control, chiefly with respect to stone free rates, but also the number of endoscopic sessions necessary for therapeutic success, the side effects, and costs.

\section{Methods}

Over a two year period 34 patients with complicated bile duct stones were prospectively and randomly enrolled in the study (Table I).

Inclusion criteria were as follows: (1) choledochal lithiasis, diagnosed by means of endoscopic retrograde cholangiography, (2) detection of the bile duct stones by abdominal ultrasound, (3) papilla within reach of the

TABLE I Patient's characteristics according to the two treatment groups

\begin{tabular}{lll}
\hline & ESWL & LISL \\
\hline Patients (n) & 17 & 17 \\
Sex & $11 \mathrm{~F}$ & $11 \mathrm{~F}$ \\
Mean age (range) (y) & $6 \mathrm{M}$ & $6 \mathrm{M}$ \\
Previous cholecystectomy (n(\%)) & $72(39-92)$ & $72(29-86)$ \\
Symptoms on admission: & $7(41)$ & $7(41)$ \\
$\quad$ Painless jaundice & 6 & 3 \\
Colic with or without icterus & 6 & 8 \\
Cholangitis & 5 & 6 \\
\hline
\end{tabular}


TABLE II Stone properties in the two treatment groups by endoscopic retrograde cholangiopancreatography radiograph

\begin{tabular}{lll}
\hline & $E S W L$ & LISL \\
\hline Stone diameter (mm) & & \\
Number of stones: & $25(8-30)$ & $24(12-38)$ \\
$\quad 1$ & 6 & 5 \\
2 & 5 & 3 \\
$\quad$ 2 & 6 & 9 \\
Stone position: & 4 & 3 \\
$\quad$ Hilum (and adjacent hepatic ducts) & 4 & 6 \\
$\quad$ Upper half of the CBD & 4 & 8 \\
$\quad$ Prepapillary half of the CBD & 9 & 5 \\
Concomitant bile duct structure & 5 & \\
\hline
\end{tabular}

*Stone diameter=maximal stone diameter in case of more than one stone.

$\mathrm{CBD}=$ Common bile duct.

endoscope, (4) failure of standard endoscopic extraction manoeuvres including endoscopic sphincterotomy and at least one attempt at mechanical lithotripsy, (5) the patient's informed consent.

Patients were excluded from the study if they had major coagulation problems, were pregnant, or if one of the inclusion criteria was not fulfilled.

All patients were diagnosed as having bile duct stones by endoscopic retrograde cholangiopancreatography at our institution or at the referring hospital. Data regarding stone size, number, and location were based on the endoscopic retrograde cholangiopancreatography radiograph (maximum stone size was measured based on the diameter of the endoscope). Table II gives the properties of the stones.

After failure of at least one attempt at mechanical lithotripsy a nasobiliary drain was inserted and the patients were randomly assigned to ESWL or LISL. The main reasons for failure of standard endoscopy were stone impaction $(n=12)$, biliary stricture $(n=8)$, or large stone diameter $(n=14)$.

All extracorporeal and intracorporeal treatment sessions were performed under intravenous sedation ( 2 to $5 \mathrm{mg}$ midazolame) and additional analgesia if necessary (25 to $100 \mathrm{mg}$ pethidine). Patients were monitored by clinical observation and continuous pulse oximetry during the procedures.

\section{METHODS}

ESWL was performed with an ultrasonically guided piezoelectric lithotripter (Piezolith 2300, R Wolf, Knittlingen, Germany). Stones were located with the two integrated $4 \mathrm{MHz}$ ultrasound scanners and the shock wave focus energy was increased from a minimum of 600 bar to the highest tolerated energy level (maximum 1200 bar). During the ESWL session the bile duct was irrigated continuously with saline solution through a nasobiliary drain.

Patients were treated with a maximum 5000 pulses per session. Fragmentation effects were controlled by abdominal ultrasound after each session. If the fragments were not properly detectable by ultrasound, fluoroscopy after instillation of contrast media via the nasobiliary drainage was used. Once stone disintegration was achieved extraction of fragments was attempted via endoscopic retrograde cholangiopancreatography. If sufficient fragmentation was not achievable after three ESWL sessions an alternative fragmentation technique was performed.

For LISL we used the Xenon flashlamp pulsed rhodamine $6 \mathrm{G}$ laser with an integrated stone tissue detection system (Lithognost, Carl Baasel Lasertechnik, Starnberg, Germany). The stone tissue detection system, as described elsewhere,${ }^{10}$ is based on the analysis of the back scattered laser energy. If the intensity of the light is below a defined threshold level, it indicates that the glass fibre is not in contact with a bile duct stone and the laser pulse is cut off with the aid of a polariser preventing tissue from being damaged. ${ }^{11}$

The laser glass fibre $(250 \mu \mathrm{m}$ or $300 \mu \mathrm{m}$ core diameter) was inserted in a $5 \mathrm{Fr}$ or $7 \mathrm{Fr}$ gauge Huibregtse or balloon catheter and passed through the papilla via the working channel of a standard duodenoscope (Olympus JF 1-T20). As the laser fibre itself is not detectable by fluoroscopy the metal tipped catheter was positioned in close proximity to the stone and then the fibre was gently pushed out of the catheter. Treatments were performed at an energy level of 100 to $150 \mathrm{~mJ}$ per pulse at a repetition rate of 8 to $10 \mathrm{~Hz}$. During the laser treatment the fragmentation effect was monitored by fluoroscopy after instillation of contrast media into the bile duct.

LISL was limited to three treatment sessions with a maximum duration of 45 minutes. Laser therapy was discontinued if there was no fragmentation effect, when the stones could not be correctly targeted by the laser fibre, or if they were not detectable by the integrated stone tissue detection system.

In the case of failure of the initial treatment patients were switched to an alternative method (LISL, ESWL, or electrohydraulic lithotripsy. For electrohydraulic lithotripsy we used the Lithotron EL-23 (Walz Electronic, Rohrdorf, Germany) under strict cholangioscopic control.

\section{STATISTICAL ANALYSES}

Data were entered into a database program and statistics were calculated with a statistical software package (Unistat 3.0 for Windows; Unistat Co, UK). Values are expressed as median (range) or mean (SD), unless otherwise stated. A Mann-Whitney $U$ test or two tailed Fisher's exact test was used for comparison between the two groups. A p value $<0.05$ was considered to be significant.

\section{Results}

ESWL GROUP

Bile duct concrements of all patients (17) were visible by the integrated ultrasound scanner before therapy. Patients were treated with a mean of $2.82(1.24)$ ESWL sessions corresponding to a mean number of 10000 (1800-19 500) shock wave pulses. To achieve complete duct clearance a mean of $1.6(0.7)$ additional sessions of endoscopic retrograde 
cholangiopancreatography were necessary. Mean duration of hospital stay was $13 \cdot 4(4 \cdot 8)$ days; mean interval between first ESWL and discharge from the hospital was $6 \cdot 2(2 \cdot 2)$ days.

In nine of the 17 patients $(53 \%)$ complete stone fragmentation using ESWL was achieved and was followed by the extraction of remaining fragments in one additional endoscopic retrograde cholangiopancreatography session. In three out of 17 patients stones were only partially fragmented; in five of the 17 patients $(29 \cdot 4 \%)$ there was no major fragmentation effect. Finally, in eight of 17 patients further fragmentation techniques were required.

The reasons for failure of ESWL therapy were as follows: loss of sonographic visibility of the bile stone during the procedure (three patients), or no fragmentation $(n=2)$, or an unsatisfactory $(n=3)$ fragmentation effect probably due to the stability of the stones.

In four patients electrohydraulic lithotripsy was used. Two of those became stone free.

In three patients fluoroscopic guided LISL was performed because of insufficient stone fragmentation during ESWL; stones were removed completely in two of these patients.

In the end, 14 of $17(82 \cdot 4 \%)$ patients were stone free after ESWL alone (nine of 17) or in combination with other methods (five of 17) in the ESWL group.

One of the patients with stones resistant to the endoscopic fragmentation techniques was sent to the surgical department to clear her bile ducts during cholecystectomy. The remaining two patients (ages 92 and 81 years) were treated by endoprosthesis insertion because they were poor candidates for operation due to coexisting diseases.

Some minor complications were noticed in the ESWL group: one patient reported pain despite intravenous sedoanalgesia; one patient had a vagovasal reaction (hypotension, dizziness) which necessitated stopping the ESWL session (the patient became stone free after the next ESWL session). One patient developed small skin petechiae immediately after ESWL.

The treatment related mortality was zero in the ESWL group.

\section{LISL GROUP}

The seventeen patients of the LISL group were treated by a mean of $1.29(0 \cdot 46)$ LISL sessions and 3744 (800-21241) correctly targeted pulses corresponding to a total number of 5300 (1440-22 831) laser pulses. The average stone detection rate (ratio of correctly targeted pulses to the total number of pulses) was about $68 \%$ with a slight but not significant increase as the study period progressed.

In combination with standard extraction manoeuvres using balloon catheters or baskets 14 of the $17(82.4 \%)$ patients became stone free with fluoroscopic guided LISL. Nine of these 14 patients were stone free in the same session; in five of them one additional endoscopic retrograde cholangiopancreatography session was necessary because the patients tolerated no further extraction manoeuvres (due to the duration of the preceeding LISL therapy). Overall, $0.65(0.68)$ additional endoscopic retrograde cholangiopancreatography sessions per patient were necessary to clear the bile ducts completely.

The average hospital stay was 12.6 (7) days in this group; the mean period between first LISL and discharge from hospital was 3.5 (3.3) days.

In three of the 17 patients LISL failed. In two of them a sufficient fibre stone contact was not achievable due to a parapapillary diverticula and an unusually dilated distal common bile duct; both patients became stone free after ultrasonically guided ESWL. In one patient only a partial stone fragmentation was achieved; he was successfully treated by mechanical lithotripsy. Combined with additional fragmentation modalities stones were removed successfully in all 17 patients $(100 \%)$ in the LISL group.

Complications due to laser therapy were as follows: one patient had an episode of acute cholangitis, and one patient complained of pain during LISL (despite intravenous analgesia). In three patients small amounts of blood were oozing from the papilla after pushing the glass fibre out of the endoscopic retrograde cholangiopancreatography catheter. However, bleeding ceased in each patient during the procedure without any intervention.

\section{COMPARISON OF ESWL WITH LISL}

Both randomly assigned groups were comparable with regard to sex, age, number, and location of the bile stones.

Number of fragmentation sessions was more favourable in the LISL group than the ESWL group (Mann-Whitney U test: $p=0 \cdot 0001$ ). The number of fragmentation pulses was significantly lower for laser fragmentation therapy $(\mathrm{p}=0.0053)$.

Additional endoscopic retrograde cholangiopancreatography sessions were necessary in both treatment groups, although significantly fewer in the LISL group (ESWL $1.6 v$ LISL $0.65)$, the difference reaching significance by Mann-Whitney U test $(p=0 \cdot 002)$.

The stone free rate according to LISL therapy alone was higher than ESWL, but this difference was not significant (Fisher's exact test, $p=0.07$ ). Using a combination of various treatment methods bile ducts were completely freed of stones in 31 of 34 patients $(91 \cdot 2 \%)$.

The two groups showed almost identical duration of hospital stay (mean 13.4 $v 12.6$ days; $p=0 \cdot 3$, NS). The interval between the first treatment session and hospital discharge was significantly shorter for those treated by laser first $(3.5 v 6.2$ days; $\mathrm{p}=0.0002)$.

An estimated cost analysis was performed based on the costs for lithotripsy sessions, the additional endoscopic retrograde cholangiopancreatography procedures, and the charges for hospital stay. The total charge to the patients in the ESWL group was $£ 4218$ and in the LISL group it was $£ 3343$; an advantage of about $£ 875$ (Table III) for laser therapy. 
TABLE III Estimated cost effectiveness of the two treatments based on the hospital charges in 1994

\begin{tabular}{lccccc}
\hline Cost item & $\begin{array}{l}\text { Costs } \\
(£)\end{array}$ & $\begin{array}{l}\text { ESWL group } \\
(\text { mean } n)\end{array}$ & $\begin{array}{l}\text { Costs } \\
(£)\end{array}$ & $\begin{array}{l}\text { LISL group } \\
(\text { mean } n)\end{array}$ & $\begin{array}{c}\text { Costs } \\
(£)\end{array}$ \\
\hline ESWL & 328 & $2 \cdot 82$ & 925 & - & - \\
LISL & 245 & - & - & $1 \cdot 29$ & 316 \\
ERCP & 82 & $1 \cdot 6^{\star}$ & 131 & $0.65^{\star}$ & $43 \cdot 3$ \\
One hospital day & 236 & $13 \cdot 4$ & 3162 & $12 \cdot 6$ & 2974 \\
Total costs per patient & & - & 4218 & - & 3343 \\
\hline
\end{tabular}

ERCP=Endoscopic retrograde cholongiopancreatography.

In this trial LISL is advantageous over ESWL with a mean benefit of about $£ 875$ per patient. ^Additional ERCP after lithotripsy.

\section{Discussion}

Interventional endoscopy has revolutionised the management of bile duct stones since the 1970s. But despite improved endoscopic equipment, standard endoscopic procedures (including sphincterotomy and mechanical lithotripsy) fail in about $5 \%$ of patients. ${ }^{4}$ For this highly selected group of patients several intracorporeal and extracorporeal fragmentation modalities have been developed. ${ }^{6} 12$

In the past most of the published findings were on the use of ESWL in the treatment of retained bile duct stones. Several types of lithotriptors with different methods of generating the shock waves were developed. Most of the ESWL treatments were performed under radiological guidance with stone free rates ranging from $53 \%$ to $94 \%$. . $^{6-8} 1314$

In our study stone disintegration through extracorporeal measures was achieved in 12 of 17 patients $(71 \%)$ but only nine of the 17 patients $(52 \cdot 4 \%)$ became stone free after ESWL alone. The number of fragmentation sessions necessary for duct clearance was higher than reported from other study groups including our previously published data using the same lithotripter. ${ }^{7}$

There are several reasons for our ESWL results being less favourable in this study. The stone properties differ between the published studies. In this trial 11 of the 17 patients treated by ESWL had two or more stones. It is known that the piezoelectric lithotriptor provides only a small focus which does not permit simultaneous fragmentation of more than one stone. ${ }^{7}$ This problem was also reported in the use of an electrohydraulic ESWL system with a small second shock wave focus. ${ }^{13}$

The loss of sonographic visualisation was another major problem. Previous studies have shown that stones in the bile duct could be detected in up to $90 \%$ by ultrasound, ${ }^{15}$ but the detection rate is lower if there are multiple stones or if stones were located in the upper third of the bile duct. ${ }^{13}$ Six patients of the ESWL group in this study had three or more stones and eight of the patients had stones in the upper half of the common bile duct or in the hilum.

The major drawback of ESWL is the time consuming approach in three steps (endoscopic retrograde cholangiopancreatography diagnosis with insertion of a nasobiliary drain, one or more treatment sessions, and finally fragment extraction by endoscopic retrograde cholangiopancreatography). The ESWL equipment is expensive with acquisition costs ranging from about $£ 270000$, but these systems are available at many institutions, because they are widely used by urologists.

Data on laser lithotripsy for complicated bile duct stones has been published from several institutions. For the coumarin green and Nd:YAG laser, stone fragmentation rates of about $80 \%$ to $90 \%$ were reported, mainly using LISL under direct cholangioscopic control. ${ }^{16-18}$ When performing LISL under fluoroscopic guidance the method failed in up to $80 \%$ of patients, in general because positioning of the glass fibre on the stone was difficult. ${ }^{17}$

The main advantage of the rhodamine laser used in this trial was the integrated stone tissue detection system. This system allows the treatment to be performed under fluoroscopic control with excellent safety and fragmentation success rates of up to $90 \% .{ }^{1019}{ }^{20}$ In this study fluoroscopic guided LISL with the rhodamine 6G laser was effective in clearing the bile ducts in about $80 \%$ of the patients of the LISL group and even in two of three patients after failure of ESWL, and there were only minor complications.

To date only two major comparative prospective and randomised studies dealing with intracorporeal and extracorporeal methods for fragmenting retained gall stones have been presented.

In a recently published trial ${ }^{21} 35$ patients with complicated bile duct stones were prospectively randomised to ESWL or cholangioscopically controlled electrohydraulic lithotripsy. The study showed a slight but not significant advantage for the electrohydraulic lithotripsy group concerning stone free rate, duration of hospital stay, and hospital charges. In the end both groups were treated comparably efficiently and safely, choledochoscopic electrohydraulic lithotripsy being advantageous considering the number of treatment sessions required.

Neuhaus et al presented a study in 1995 in which radiologically guided ESWL was compared prospectively with laser lithotripsy. ${ }^{22}$ Although using the rhodamine $6 \mathrm{G}$ laser with the automatic stone tissue detection system almost all laser therapies ( 28 out of 30 patients) were performed under cholangioscopic control and most of the patients were treated via the percutaneous route. LISL was preferable and superior to ESWL under these conditions for the number of treatment sessions $(p<0.01)$ and the duration of treatment $(p<0.0001)$. The stone free rate differed significantly in these circumstances (ESWL 22/30 v LISL 29/30; $\mathrm{p}<0.05$ ).

As stated above we only found a tendency for fluoroscopic guided ("blind") LISL to be more efficient for duct clearance than sonographic controlled ESWL. This did not reach significance $(p=0.07)$, probably due to the few patients enrolled into the study.

In the LISL group the number of treatment sessions required was significantly lower $(p=0.0001)$ and even the number of additional sessions of endoscopic retrograde cholangiopancreatography after the fragmentation was 
favourably less $(p=0 \cdot 002)$. This is very similar to the two previous studies mentioned above.

By contrast with the study of Neuhaus $e t a l^{22}$ in our trial laser fragmentation was performed exclusively under fluoroscopic guidance. This manner of treatment was chosen due to the cost effeciveness of using a cholangioscopic control. In our opinion the future for intracorporeal laser lithotripsy is the fluoroscopically guided approach. This reduces the costs for the endoscopic equipment (for example, the babyscope) and can be performed by one experienced gastroenterologist.

Cost analysis of our study was estimated, comprising direct hospital charges and costs of endoscopy and lithotripsy. The estimation was based on our specific local charges and did not include the costs to society (for example, lost wages and productivity), which is reasonable as most of our study patients were over 65 years old and retired. Despite a comparable total duration of hospital stay, laser therapy is advantageous over ESWL with a mean benefit of about $£ 875$ per patient. These charges are representative for our hospital and may not be correct for other institutions and countries.

In conclusion, laser lithotripsy with a stone tissue recognition system was at least as efficient as ESWL in fragmenting retained bile duct stones with a tendency towards a higher stone free rate $(p=0.07)$. When performing laser therapy as a first line method, significantly less fragmentation sessions and additional endoscopic retrograde cholangiopancreatography sessions were necessary to achieve complete bile duct clearance. The lower number of therapeutic interventions resulted in a better cost effectiveness for LISL. As stated, complete duct clearance can be reached more rapidly and less expensively by fluoroscopic guided LISL than by ESWL under sonographic guidance and LISL should be considered as the first line treatment for retained bile duct stones.

This work was presented in part at the 96th Annual Meeting of the American Gastroenterological Association/Digestive Disease Week, San Francisco, California, 1996. We are gratefu to Miss Eibhlin Ni Choileáin for her help in preparing thi manuscript.

1 Classen M, Demling L. Endoskopische Sphinkterotomie der Papilla Vateri und Steiner choledochus. Dtsch Med Wochenschr 1974; 99: 496-7.
2 Kawai K, Akasaka Y, Murakami K. Endoscopic sphincterotomy of the ampulla of Vater. Gastrointest Endosc 1974; 20: 148-51.

3 Escourrou J, Berthélemy P. Biliary complications after laparoscopic cholecystectomy. Dtsch Med Wochenschr 1993; 118: 1157-62

4 Classen M, Hagenmüller F, Knyrim K, Frimberger E. Giant bile duct stones - non-surgical treatment. Endoscopy 1988; 20: 21-6.

5 Schneider MU, Matek W, Bauer R, Domschke W. Mechanical lithotripsy of bile duct stones in 209 patients: effects of technical advances. Endoscopy 1988; 20: 248-53.

6 Binmoeller KF, Bruckner M, Thonke F, Soehendra N Treatment of difficult bile duct stones using mechanical, electrohydraulic and extracorporeal shock wave lithotripsy. Endoscopy 1993; 25: 201-6.

7 Adamek HE, Buttmann A, Hartmann CM, Jakobs R, Riemann JF. Extracoporeal piezoelectric lithotripsy of intra- and extrahepatic biliary tract stones. Dtsch Med Wochenschr 1993; 118: 1053-9.

8 Sauerbruch T, Stern M. Fragmentation of bile duct stones by extracorporeal shock waves. Gastroenterology 1989; 96: $146-51$

9 Prat F, Fritsch J, Choury AD, Frouge C, Marteau V, Etienne JP. Laser lithotripsy of difficult biliary stones Gastrointest Endosc 1994; 40: 290-5.

10 Ell C, Hochberger J, May A, Fleig WE, Bauer R, Mendez L, Hahn EG. Laser lithotripsy of difficult bile duct stones by means of a rhodamine- $6 \mathrm{G}$ laser and an integrated automatic stone-tissue detection system. Gastrointest Endosc 1993; 39: 755-62.

11 Schmeller N, Kriegmair M, Liedl B, Hofstetter A Muschter R, Thomas S, Knipper A. Laserlithotripsie mit automatischer Abschaltung bei Gewebekontakt. Urologe $A$ 1990; 29: 309-12.

12 Classen $M$, Hagenmueller $F$. Treatment of stones in the bile duct via duodenoscopy. Endoscopy 1989; 21: 375-7.

13 Ponchon T, Martin X, Barkun A, Mestat JL, Chavaillon A, Boustière C. Extracorporeal lithotripsy of bile duct stones using ultrasonography for stone localization. Gastroenterology 1990; 98: 726-32.

14 Sauerbruch T, Holl J, Sackmann M, Paumgartner G. Fragmentation of bile duct stones by extracorporeal shock wave lithotripsy: a five-year experience. Hepatology 1992 15: $208-14$

15 Wermke W. Sonographic diagnosis of concrements of the bile ducts. Ultraschall Med 1992; 13: 246-54

16 Nishioka NS, Levins PC, Murray SC, Parrish JA, Anderson RR. Fragmentation of biliary calculi with tunable dye lasers. Gastroenterology 1987; 93: 250-5.

17 Cotton PB, Kozarek RA, Schapiro RH, Nishioka NS Kelsey PB, Ball TJ, et al. Endoscopic laser lithotripsy of Kelsey PB, Ball TJ, et al. Endoscopic laser lithotripsy of large bile
$1128-33$.

18 Kozarek RA, Low DE, Ball TJ. Tunable dye laser lithotripsy: in vitro studies and in vivo treatment of choledocholithiasis. Gastrointest Endosc 1988; 34 418-22.

19 Neuhaus H, Hoffmann W, Gottlieb K, Classen M Endoscopic lithotripsy of bile duct stones using a new laser with automatic stone recognition. Gastrointest Endosc 1994; 40: 708-15.

20 Jakobs R, Maier M, Kohler B, Riemann JF. Peroral laser lithotripsy of difficult intrahepatic and extrahepatic bile duct stones. Laser effectiveness using an automatic stonetissue discrimination system. Am F Gastroenterol 1996; 91: tissue discri

21 Adamek HE, Buttmann A, Wessbecher R, Kohler B, Riemann JF. Clinical comparison of extracorporeal piemann JF. Clinical comparison of extracorporeal piezoelectric lithotripsy (EPL) and intracorporeal electrohydraulic lithotripsy (EHL) in

22 Neuhaus H, Zillinger C, Born P, Ott R, Allescher H, Rösch T, Classen M. Randomized study of endoscopic laserlithotripsy (ELL) $v$ extracorporeal shock-wave lithotripsy (ESWL) for difficult bile duct stones Endoscopy 1995; 27: S48(A) 